Real Analysis Exchange

Vol. 24(2), 1998/9, pp. 567-578

H. Hudzik and M. Mastylo*, Faculty of Mathematics and Computer Science, A. Mickiewicz University, Matejki 48/49, 60-769 Poznan, Poland, e-mail: hudzik@math.amu.edu.pl and mastylo@math.amu.edu.pl

L. Maligranda ${ }^{\dagger}$ and L. E. Persson, Department of Mathematics, Luleå University of Technology, S-971 87 Luleå, Sweden,

e-mail: lech@sm.luth.se and larserik@sm.luth.se

\title{
EXTENSION OF SUBMULTIPLICATIVITY AND SUPERMULTIPLICATIVITY OF ORLICZ FUNCTIONS
}

\begin{abstract}
Results concerning extension of submultiplicativity and supermultiplicativity for Orlicz functions are proved. A typical result is the following: If the Orlicz function $\varphi$ is submultiplicative at infinity, then an Orlicz function $\psi$, which is submultiplicative on $\mathbb{R}_{+}$, equivalent to $\varphi$ at infinity and satisfying $\psi(u) / u \rightarrow 0$ as $u \rightarrow 0$ exists if and only if the conjugate function $\varphi^{*}$ satisfies the $\Delta_{2}$-condition at infinity. Some complementary results and (counter-)examples are also included.
\end{abstract}

\section{Introduction}

The Orlicz functions are parameters generating Orlicz spaces, which are generalizations of the well known $L_{p}$ spaces. An $L_{p}$ space is generated by the power function $\varphi(u)=u^{p}$ for $u \geq 0$, which is both submultiplicative and supermultiplicative on $[0, \infty)$ for any $0<p<\infty$. Submultiplicative as well as supermultiplicative functions have found interesting applications in the study of many different problems (see e.g. [8], [10], [4], [5]). Note that there is a strict connection between convex and concave functions. Concave functions play a fundamental role in interpolation theory. They parameterize the interpolation methods. For example, the submultiplicative concave function

Key Words: Orlicz functions, convex functions, conjugate functions, submultiplicative functions, supermultiplicative functions, extensions

Mathematical Reviews subject classification: 26A09, 26A48, 26A51, 26D20

Received by the editors March 23, 1998

* Research supported by KBN-Grant 2 PO3A 05009

${ }^{\dagger}$ Research partially supported by NFR-Grant 06857-306 
$f(x)=\ln \left(e^{2}+x\right)$ on $[0, \infty)$ was used by Zafran [14] to produce an interpolation Banach algebra, which gives a negative solution of the dichotomy problem for homogeneous algebras. Moreover, the submultiplicative function $f(x)=\log _{2}(1+x)$ was used by Odell and Schlumprecht in their remarkable paper [11] when they solve the distortion problem for Hilbert spaces. This shows surprisingly deep application of submultiplicative functions to the construction of new Banach spaces. We note that in the book [8], as well as many other works, submultiplicative and supermultiplicative functions are investigated and applied.

The present paper is in a sense a continuation of the paper [7], where the following extension theorems were proved, answering a question posed by Krasnoselskii and Rutickii [8], p. 30.

Theorem A. ([7], Th. 2). Let $\varphi$ be an Orlicz function, which is submultiplicative at infinity. If the conjugate function $\varphi^{*}$ satisfies the $\Delta_{2}$-condition at infinity, then we can find an Orlicz function $\psi$ which is submultiplicative on $\mathbb{R}_{+}$, equivalent to $\varphi$ at infinity and satisfying $\psi(u) / u \rightarrow 0$ as $u \rightarrow 0^{+}$.

Theorem B. ([7], Th. 3). Let $\varphi$ be an Orlicz function, which is submultiplicative at zero. Then an Orlicz function $\psi$, which is submultiplicative on $\mathbb{R}_{+}$, equivalent to $\varphi$ at zero and satisfying that $\psi(u) / u \rightarrow \infty$ as $u \rightarrow \infty$ exists if and only if $\varphi$ satisfies the $\Delta_{2}$-condition at zero.

In this paper we will prove that Theorem A is in fact sharp in the sense that the implication there can be replaced by an equivalence (see Theorem 1). We also prove some corresponding extension results when the Orlicz function $\varphi$ is supermultiplicative at infinity (Theorem 1) or supermultiplicative at zero (Theorem 2). These results are proved in Section 3. Some complementary results, remarks and (counter-)examples can be found in Section 4.

\section{Notation, Definitions and Remarks}

We collect now some necessary notation, definitions and remarks.

By $\varphi$ we denote an Orlicz function; i.e., a function from $\mathbb{R}_{+}=[0,+\infty)$ into $\mathbb{R}_{+}$, which is convex and vanishing only at zero. By $\varphi^{*}$ we denote the function conjugate to $\varphi$, which is defined for $u \geq 0$ by $\varphi^{*}(u)=\sup \{u v-\varphi(v): v \geq 0\}$. It is well known that $\varphi^{*}$ vanishes only at zero if and only if $\varphi(u) / u \rightarrow 0$ as $u \rightarrow 0^{+}$and $\varphi^{*}$ has finite values if and only if $\varphi(u) / u \rightarrow \infty$ as $u \rightarrow \infty$. An Orlicz function $\varphi$ such that $\varphi(u) / u \rightarrow 0$ as $u \rightarrow 0^{+}$and $\varphi(u) / u \rightarrow \infty$ as $u \rightarrow \infty$ is called an $N$-function (see [8]).

We say that an Orlicz function $\varphi$ satisfies the $\Delta_{2}$-condition at zero (at infinity) if there are positive constants $K$ and $u_{0}$ such that $\varphi(2 u) \leq K \varphi(u)$ holds for 
all $u \in\left[0, u_{0}\right]\left(u \in\left[u_{0}, \infty\right)\right)$. If the above inequality on $\varphi$ holds for all $u \in \mathbb{R}_{+}$, then we say that $\varphi$ satisfies the $\Delta_{2}$-condition on $\mathbb{R}_{+}$.

We say that an Orlicz function $\varphi$ is submultiplicative (supermultiplicative) at zero, at infinity if there exist positive constants $L$ and $u_{0}$ such that

$$
\varphi(u v) \leq L \varphi(u) \varphi(v) \quad(\text { respectively } \quad \varphi(u v) \geq L \varphi(u) \varphi(v))
$$

holds for all $u, v$ in $\left[0, u_{0}\right]$ or $\left[u_{0}, \infty\right)$, respectively. If the above inequality holds for all $u, v \in \mathbb{R}_{+}$, then we say that $\varphi$ is submultiplicative (supermultiplicative) on $\mathbb{R}_{+}$.

It is easy to see that submultiplicativity at infinity implies the $\Delta_{2}$-condition at infinity, supermultiplicativity at zero implies the $\Delta_{2}$-condition at zero, and that submultiplicativity as well as supermultiplicativity on $\mathbb{R}_{+}$imply the $\Delta_{2^{-}}$ condition on $\mathbb{R}_{+}$. Our Example 3 shows, in particular, that none of the above implications hold in the reversed direction.

Remark 1. The condition that $\varphi$ is supermultiplicative at infinity (submultiplicative at zero) does not guarantee that $\varphi$ satisfies the $\Delta_{2}$-condition at infinity $\left(\Delta_{2}\right.$-condition at zero). See Example 1 .

We say that two Orlicz functions $\varphi$ and $\psi$ are equivalent at zero (at infinity) if there are positive constants $C_{1}, C_{2}$ and $u_{0}$ such that the inequalities $\varphi\left(C_{1} u\right) \leq \psi(u) \leq \varphi\left(C_{2} u\right)$ hold for all $u \in\left[0, u_{0}\right]$ (for all $u \in\left[u_{0}, \infty\right)$ ). If the above inequalities on $\varphi$ and $\psi$ hold for all $u \in \mathbb{R}_{+}$, then we say that $\varphi$ and $\psi$ are equivalent on $\mathbb{R}_{+}$.

Remark 2. If two Orlicz functions $\varphi$ and $\psi$ satisfy $\varphi(u) \leq \psi(C u)$ for all $u \geq u_{0}$, then $\psi^{*}(u / C) \leq \varphi^{*}(u)$ for all $u \geq u^{*}$, where $u^{*}$ is sufficiently large or $u^{*}=0$ if $u_{0}=0$ (see [8, p. 16] or [10, p. 49] or [12, p. 15]). Similarly, if $\varphi(u) \leq \psi(C u)$ for all $0<u \leq u_{0}$, then $\psi^{*}(u / C) \leq \varphi^{*}(u)$ for all $0<u \leq u^{*}$ with $u^{*}$ sufficiently small. In particular, two Orlicz functions $\varphi$ and $\psi$ are equivalent at infinity (at zero or on $\mathbb{R}_{+}$) if and only if, their conjugate functions $\varphi^{*}$ and $\psi^{*}$ are equivalent at infinity (at zero or on $\mathbb{R}_{+}$).

\section{$3 \quad$ Extension Results}

Our main tool in the proofs is the following lemma.

Lemma 1. Let $\varphi$ be an Orlicz function and let the function $\psi$ be defined by

$$
\psi(u)=\int_{0}^{u} \frac{1}{t \varphi\left(\frac{1}{t}\right)} d t
$$


(a) Then $\psi$ is also an Orlicz function. Moreover, $\varphi$ satisfies the $\Delta_{2}$-condition at zero (at infinity) $\left[\right.$ on $\left.\mathbb{R}_{+}\right]$if and only if $\psi$ satisfies the $\Delta_{2}$-condition at infinity (at zero) $\left[\right.$ on $\left.\mathbb{R}_{+}\right]$.

(b) The function $\varphi$ is submultiplicative at infinity (on $\mathbb{R}_{+}$) if and only if the function $\psi$ is supermultiplicative at zero (on $\mathbb{R}_{+}$). The function $\varphi$ is supermultiplicative at zero (on $\mathbb{R}_{+}$) if and only if the function $\psi$ is submultiplicative at infinity (on $\mathbb{R}_{+}$).

Moreover, also for the remaining two cases (submultiplicativity at zero and supermultiplicativity at infinity) the equivalences in (b) hold if we add the assumption that $\varphi$ (or $\psi$ ) satisfies the corresponding $\Delta_{2}$-condition.

Proof. (a) Since $\frac{1}{t \varphi\left(\frac{1}{t}\right)}$ is an increasing function on $(0, \infty)$ it follows that $\psi$ is an Orlicz function. Moreover, for all $u>0$

$$
\frac{1}{2 \varphi\left(\frac{2}{u}\right)} \leq \psi(u) \leq \frac{1}{\varphi\left(\frac{1}{u}\right)}
$$

and

$$
\psi(u)+\frac{1}{\varphi\left(\frac{1}{u}\right)} \leq \psi(2 u) \leq \psi(u)+\frac{1}{2 \varphi\left(\frac{1}{2 u}\right)} .
$$

If $\varphi$ satisfies the $\Delta_{2}$-condition at infinity, i.e., if there are $K>0$ and $u_{0}>0$ such that $\varphi(2 v) \leq K \varphi(v)$ for all $v \geq u_{0}$, then, for all $u \in\left(0, \frac{u_{0}}{2}\right]$,

$$
\varphi\left(\frac{2}{u}\right) \leq K \varphi\left(\frac{1}{u}\right) \leq K^{2} \varphi\left(\frac{1}{2 u}\right) .
$$

Thus

$$
\begin{aligned}
\psi(2 u) & \leq \psi(u)+\frac{1}{2 \varphi\left(\frac{1}{2 u}\right)} \leq \psi(u)+\frac{K^{2}}{2} \frac{1}{\varphi\left(\frac{2}{u}\right)} \\
& \leq \psi(u)+\frac{K^{2}}{2} \int_{\frac{u}{2}}^{u} \frac{1}{t \varphi\left(\frac{1}{t}\right)} d t \leq\left(\frac{K^{2}}{2}+1\right) \psi(u)
\end{aligned}
$$

for all $u \in\left(0, u_{0} / 2\right]$; i.e., $\psi$ satisfies the $\Delta_{2}$-condition at zero.

Assume now that $\psi$ satisfies the $\Delta_{2}$-condition at zero; i.e., there is $K>1$ and $u_{0}>0$ such that $\psi(2 v) \leq K \psi(v)$ for all $v \in\left(0, u_{0}\right]$. Then, for all $u \geq 2 / u_{0}$,

$$
\psi\left(\frac{1}{u}\right)+\frac{1}{\varphi(u)} \leq \psi\left(\frac{2}{u}\right) \leq K \psi\left(\frac{1}{u}\right) .
$$

Therefore

$$
\varphi(2 u) \leq \frac{1}{\psi\left(\frac{1}{2 u}\right)} \leq \frac{K}{\psi\left(\frac{1}{u}\right)} \leq \frac{K}{K-1} \varphi(u)
$$


for all $u \geq 2 / u_{0}$; i.e., $\varphi$ satisfies the $\Delta_{2}$-condition at infinity. We can prove the second case in a similar fashion.

(b) If $\varphi$ is submultiplicative at infinity, then $\varphi$ satisfies the $\Delta_{2}$-condition at infinity. By using (2), for $u, v>0$ small enough, we obtain

$$
\psi(u) \psi(v) \leq \frac{L}{\varphi\left(\frac{1}{u v}\right)} \leq \frac{K L}{\varphi\left(\frac{2}{u v}\right)} \leq 2 K L \psi(u v),
$$

which means that $\psi$ is supermultiplicative at zero.

On the other hand, if $\psi$ is supermultiplicative at zero, then $\psi$ satisfies the $\Delta_{2}$-condition at zero. Thus, by again using (2), we find that for $u$ and $v$ sufficiently large

$$
\varphi(u v) \leq \frac{1}{L \psi\left(\frac{1}{u}\right) \psi\left(\frac{1}{v}\right)} \leq \frac{4}{L} \varphi(2 u) \varphi(2 v) \leq \frac{4 K^{2}}{L} \varphi(u) \varphi(v),
$$

so that $\varphi$ is submultiplicative at infinity. The other cases can be proved similarly.

Now we state the announced more precise version of Theorem A together with the supermultiplicative version.

\section{Theorem 1.}

(a) Let $\varphi$ be an Orlicz function which is submultiplicative at infinity. The following conditions are equivalent.

(i) There exists an Orlicz function $\psi$, which is submultiplicative on $\mathbb{R}_{+}$, equivalent to $\varphi$ at infinity and satisfying that $\psi(u) / u \rightarrow 0$ as $u \rightarrow 0^{+}$.

(ii) $\varphi^{*}$ satisfies the $\Delta_{2}$-condition at infinity.

(b) Let $\varphi$ be an Orlicz function which is supermultiplicative at infinity. The following conditions are equivalent.

(i) There exists an Orlicz function $\Psi$ which is supermultiplicative on $\mathbb{R}_{+}$ and equivalent to $\varphi$ at infinity.

(ii) $\varphi$ satisfies the $\Delta_{2}$-condition at infinity.

Proof. (a) The implication (ii) $\Rightarrow$ (i) is nothing else but Theorem A. Assume now that (i) holds. In particular, this means that there exists a constant $L>0$ such that $\psi(u v) \leq L \psi(u) \psi(v) \quad$ for all $u, v>0$. Moreover, since $\psi(u) / u \rightarrow 0$ as $u \rightarrow 0$, there exists $a \in(0,1)$ such that $\psi(a) / a<1 /(2 L)$. Therefore

$$
\psi(a v) \leq L \psi(a) \psi(v) \leq \frac{a}{2 L} L \psi(v)=\frac{a}{2} \psi(v) .
$$


Hence,

$$
\begin{aligned}
\psi^{*}(2 u) & =\sup _{v>0}\{2 u v-\psi(v)\} \leq \sup _{v>0}\left\{2 u v-\frac{2}{a} \psi(a v)\right\} \\
& =\frac{2}{a} \sup _{v>0}\{u a v-\psi(a v)\}=\frac{2}{a} \psi^{*}(u) .
\end{aligned}
$$

This means that $\psi^{*}$ satisfies the $\Delta_{2}$-condition on $\mathbb{R}_{+}$. Moreover, by the assumption, $\varphi$ is equivalent to $\psi$ at infinity which gives, by $\operatorname{Remark} 2$, that $\varphi^{*}$ is equivalent to $\psi^{*}$ at infinity. Therefore, $\varphi^{*}$ satisfies the $\Delta_{2}$-condition at infinity.

(b) (i) $\Rightarrow$ (ii) The function $\Psi$ is supermultiplicative on $\mathbb{R}_{+}$and therefore it satisfies the $\Delta_{2}$-condition on $\mathbb{R}_{+}$. Moreover, $\varphi$ as a function equivalent to $\Psi$ at infinity satisfies the $\Delta_{2}$-condition at infinity.

(ii) $\Rightarrow$ (i) Assume that $\varphi$ is supermultiplicative at infinity and satisfies the $\Delta_{2^{-}}$ condition at infinity. Let $\psi$ be defined as in Lemma 1 . According to this lemma we know that $\psi$ is submultiplicative at zero and satisfies the $\Delta_{2}$-condition at zero. In view of Theorem $\mathrm{B}$, these two properties of $\psi$ imply the existence of an Orlicz function $\Phi$ which is equivalent to $\psi$ at zero and submultiplicative on $\mathbb{R}_{+}$(and also $\Phi(u) / u \rightarrow \infty$ as $\left.u \rightarrow \infty\right)$. However, again according to Lemma 1, the submultiplicativity of $\Phi$ on $\mathbb{R}_{+}$is equivalent to the supermultiplicativity on $\mathbb{R}$ of the function $\Psi$ defined by

$$
\Psi(u)=\int_{a}^{u} \frac{1}{t \Phi\left(\frac{1}{t}\right)} d t .
$$

It only remains to prove that $\Psi$ is equivalent to $\varphi$ at infinity.

We know that $\Phi$ is equivalent to $\psi$ at zero; i.e., there are positive constants $C_{1}, C_{2}$ and $u_{0}$ such that $\Phi\left(C_{1} u\right) \leq \psi(u) \leq \Phi\left(C_{2} u\right)$ for all $u \in\left(0, u_{0}\right]$. By using inequalities (2) to both functions $\psi$ and $\Psi$ we obtain, for $u$ large enough,

$$
\Psi(u) \leq \frac{1}{\Phi\left(\frac{1}{u}\right)} \leq \frac{1}{\psi\left(\frac{1}{C_{2} u}\right)} \leq 2 \varphi\left(2 C_{2} u\right)
$$

and

$$
\Psi(u) \geq \frac{1}{2 \Phi\left(\frac{2}{u}\right)} \geq \frac{1}{\psi\left(\frac{2}{C_{1} u}\right)} \geq 2 \varphi\left(\frac{C_{1} u}{2}\right),
$$

which means that $\Psi$ is equivalent to $\varphi$ at infinity. Note also that $\Psi(u) / u \rightarrow \infty$ as $u \rightarrow \infty$ iff $\varphi(u) / u \rightarrow \infty$ as $u \rightarrow \infty$.

We also have the following result for the case when $\varphi$ is a supermultiplicative function at zero. 
Theorem 2. Let $\varphi$ be an Orlicz function, which is supermultiplicative at zero. Then:

(a) There exists an Orlicz function $\psi_{1}$, which is supermultiplicative on $\mathbb{R}_{+}$ and equivalent to $\varphi$ at zero.

(b) The following conditions are equivalent:

(i) There exists an Orlicz function $\psi_{2}$, which is supermultiplicative on $\mathbb{R}_{+}$, equivalent to $\varphi$ at zero and satisfying $\psi_{2}(u) / u \rightarrow \infty$ as $u \rightarrow \infty$.

(ii) $\varphi^{*}$ satisfies the $\Delta_{2}$-condition at zero.

Proof. (a) If $\varphi$ is an Orlicz function which is supermultiplicative at zero, then $\psi$ defined as in Lemma 1 is an Orlicz function which is submultiplicative at infinity. Now, according to Remark 2 in [7], there exists an Orlicz function $\Phi$, which is equivalent to $\psi$ at infinity and submultiplicative on $\mathbb{R}_{+}$. By Lemma 1 the function

$$
\psi_{1}(u)=\int_{0}^{u} \frac{1}{t \Phi\left(\frac{1}{t}\right)}, d t
$$

is supermultiplicative on $\mathbb{R}_{+}$and, exactly as in the proof of Theorem 1 (b), we can find that the function $\psi_{1}$ is equivalent to $\varphi$ at zero. The proof is complete.

(b) Assume that (i) is satisfied. Let $a \in(0,1)$ be such that $a \psi_{2}(1 / a) \geq 2 / L$, where $L$ is a positive constant from the assumption $\psi_{2}(u v) \geq L \psi_{2}(u) \psi_{2}(v)$ for all $u, v \in \mathbb{R}_{+}$. Then, for all $u>0$, we have

$$
\psi_{2}(a u) \leq \frac{1}{L \psi_{2}\left(\frac{1}{a}\right)} \psi_{2}(u) \leq \frac{a}{2} \psi_{2}(u) .
$$

Therefore, as in the proof of Theorem 1 (a), we find that $\psi_{2}^{*}$ satisfies the $\Delta_{2}$ condition on $\mathbb{R}_{+}$. Moreover, since $\psi_{2}$ is equivalent to $\varphi$ at zero we find that $\psi_{2}^{*}$ is equivalent to $\varphi^{*}$ at zero (cf. Remark 2) and so $\varphi^{*}$ satisfies the $\Delta_{2}$-condition at zero which means that (ii) holds.

Conversely, suppose that (ii) is valid, i.e., $\varphi$ is submultiplicative at zero and $\varphi^{*}$ satisfies the $\Delta_{2}$-condition at zero. Let $\psi$ be defined as in Lemma 1 .

First we prove that $\psi^{*}$ satisfies the $\Delta_{2}$-condition at infinity. Without loss of generality we can assume that the derivative $\varphi^{\prime}$ is continuous. (Otherwise we take the function $\varphi_{1}(u)=\int_{0}^{u} \frac{\varphi(t)}{t} d t$, which is an Orlicz function with a continuous derivative and which is equivalent to $\varphi$ on $\mathbb{R}_{+}$, because $\varphi_{1}(u / 2) \leq$ $\varphi(u) \leq \varphi_{1}(u)$ for all $u>0$.) Then, $\frac{u \psi^{\prime}(u)}{\psi(u)}=\frac{1}{\psi(u) \varphi\left(\frac{1}{u}\right)}$ and, by l'Hôpital 
rule,

$$
\liminf _{u \rightarrow \infty} \frac{\frac{1}{\varphi\left(\frac{1}{u}\right)}}{\psi(u)}=\liminf _{u \rightarrow \infty} \frac{\varphi^{\prime}\left(\frac{1}{u}\right)}{\psi^{\prime}(u) u^{2}\left[\varphi\left(\frac{1}{u}\right)\right]^{2}}=\liminf _{u \rightarrow \infty} \frac{\varphi^{\prime}\left(\frac{1}{u}\right)}{u \varphi\left(\frac{1}{u}\right)}=\liminf _{v \rightarrow 0^{+}} \frac{v \varphi^{\prime}(v)}{\varphi(v)} .
$$

Thus

$$
\liminf _{u \rightarrow \infty} \frac{u \psi^{\prime}(u)}{\psi(u)}=\liminf _{v \rightarrow 0^{+}} \frac{v \varphi^{\prime}(v)}{\varphi(v)} .
$$

Now according to Theorem 4.3 in Krasnoselskii-Rutickii's book [8] (see also [12], p. 26), $\varphi^{*}$ satisfies the $\Delta_{2}$-condition at zero if and only if the limit on the right hand side of the above expression is bigger that some number $\alpha>1$. (In the book only a version at infinity is proved but the similar result is obviously also true at zero.) The above equality shows that then the limit on the left hand side of the above expression is bigger than some $\alpha>1$, and again by Theorem 4.3 in [8], this means that $\psi^{*}$ satisfies the $\Delta_{2}$-condition at infinity. Therefore $\psi$ is submultiplicative at infinity and $\psi^{*}$ satisfies the $\Delta_{2}$-condition at infinity. By using Theorem A we conclude that there exists an Orlicz function $\psi_{1}$, which is submultiplicative on $\mathbb{R}_{+}$, equivalent to $\psi$ at infinity and such that $\psi_{1}(u) / u \rightarrow 0$ as $u \rightarrow 0^{+}$. Then the function $\psi_{2}$ defined by

$$
\psi_{2}(u)=\int_{0}^{u} \frac{1}{t \psi_{1}\left(\frac{1}{t}\right)} d t
$$

is supermultiplicative on $\mathbb{R}_{+}$, equivalent to $\varphi$ at zero (More precisely, $\psi_{2}(u)$ is equivalent to $\frac{1}{\psi_{1}\left(\frac{1}{u}\right)}$ for all $u>0, \psi_{1}$ is equivalent to $\psi$ for large $u$ and $1 / \psi\left(\frac{1}{u}\right)$ is equivalent to $\varphi(u)$ for all $u>0$.) and satisfies $\psi_{2}(u) / u \rightarrow \infty$ as $u \rightarrow \infty$.

Remark 3. The proofs of the implication (ii) $\Rightarrow$ (i) in Theorem 1 (a) and Theorem 2 (b) show the following property. If $\varphi$ is an $N$-function, which is either submultiplicative on $\mathbb{R}_{+}$or supermultiplicative on $\mathbb{R}_{+}$, then both $\varphi$ and $\varphi^{*}$ satisfy the $\Delta_{2}$-condition on $\mathbb{R}_{+}$.

\section{Complementary Results, Remarks and Examples}

First we present the following complement of the statements in Remark 1.

\section{Example 1.}

(a) [7, Ex. 2] There exists an Orlicz function $\varphi$ such that $\varphi(u) / u \rightarrow 0$ as $u \rightarrow 0^{+}, \varphi$ is submultiplicative at zero but $\varphi$ does not satisfy the $\Delta_{2^{-}}$ condition at zero. 
(b) There exists an Orlicz function $\psi$ such that $\psi(u) / u \rightarrow \infty$ as $u \rightarrow \infty$, $\psi$ is supermultiplicative at infinity, but $\psi$ does not satisfy the $\Delta_{2}$-condition at infinity.

In fact, for $\alpha>0$ we consider $\varphi_{\alpha}$ defined by $\varphi_{\alpha}(0)=0$ and

$$
\varphi_{\alpha}(u)=u^{1+\alpha} \ln \left(1+e^{-\frac{1}{u^{\alpha}}}\right) \text { for } u>0 .
$$

It has been proved in [7] that $\varphi_{\alpha}$ has the properties from (a): $\varphi_{\alpha}(u) / u \rightarrow 0$ as $u \rightarrow 0^{+}, \varphi_{\alpha}$ is submultiplicative on $[0,1]$ and $\varphi_{\alpha}$ does not satisfy the $\Delta_{2^{-}}$ condition at zero. Therefore, according to Lemma 1, the function

$$
\psi_{\alpha}(u)=\int_{0}^{u} \frac{1}{t \varphi_{\alpha}\left(\frac{1}{t}\right)} d t
$$

has the desired properties in (b).

Remark 4. Ando [2, Th. 1] (cf. also [12], pp. 28-29) proved the following duality property. Let $\varphi$ be an $N$-function. Then $\varphi$ is submultiplicative at infinity if and only if $\varphi^{*}$ is "almost" supermultiplicative at infinity, i.e., $\varphi^{*}$ satisfies $\varphi^{*}(u) \varphi^{*}(v) \leq \varphi^{*}\left(L^{\prime} u v\right)$ for some $L^{\prime}>0$ and for large $u, v$.

Of course, repeating his proof, we can show that in the class of $N$-functions the following holds. $\varphi$ is submultiplicative on $\mathbb{R}_{+}$if and only if $\varphi^{*}$ is supermultiplicative on $\mathbb{R}_{+}$.

In our next example we illustrate the fact that the difference between the case at infinity and the case on $\mathbb{R}_{+}$is really essential.

Example 2. The functions

$$
\varphi(u)=(1+u) \ln (1+u)-u \quad \text { and } \quad \varphi(u)=u \ln (1+u)
$$

are examples of $N$-functions, which are submultiplicative at infinity and $\varphi^{*}$ do not satisfy the $\Delta_{2}$-condition at infinity.

In view of Remark 3, it is natural to ask the question if, for any Orlicz function $\varphi$ such that both $\varphi$ and $\varphi^{*}$ satisfy the $\Delta_{2}$-condition on $\mathbb{R}_{+}$there exists an Orlicz function $\psi$ which is equivalent to $\varphi$ on $\mathbb{R}_{+}$and such that $\psi$ is either submultiplicative on $\mathbb{R}_{+}$or supermultiplicative on $\mathbb{R}_{+}$. The negative answer of this question is given in our next example.

\section{Example 3.}

(a) There exists an Orlicz function $\varphi$ such that both $\varphi$ and $\varphi^{*}$ satisfy the $\Delta_{2}$-condition on $\mathbb{R}_{+}$and $\varphi(u) / u \rightarrow 0$ as $u \rightarrow 0^{+}$but $\varphi$ is not equivalent to any Orlicz function $\psi$ which is submultiplicative on $\mathbb{R}_{+}$. 
(b) There exists an Orlicz function $\psi$ such that both $\psi$ and $\psi^{*}$ satisfy the $\Delta_{2}$-condition on $\mathbb{R}_{+}$and $\psi(u) / u \rightarrow \infty$ as $u \rightarrow \infty$, but $\psi$ is not equivalent to any Orlicz function $\Phi$ which is supermultiplicative on $\mathbb{R}_{+}$.

First we observe that if $\varphi$ is an Orlicz function equivalent to another Orlicz function $\psi$, which is submultiplicative on $\mathbb{R}_{+}$, then $\varphi$ is equivalent to $\bar{\varphi}$ on $\mathbb{R}_{+}$, where $\bar{\varphi}$ is defined by $\bar{\varphi}(u):=\sup _{v>0} \frac{\varphi(u v)}{\varphi(v)}$. Now, consider the Orlicz function $\varphi(u)=u^{p} \ln (1+u)$ for $u \geq 0$, where $1<p<\infty$. Then $\varphi(u) / u \rightarrow 0$ as $u \rightarrow 0^{+}$ and both $\varphi$ and $\varphi^{*}$ satisfy the $\Delta_{2}$-condition on $\mathbb{R}_{+}$. The function $\varphi$ is not equivalent to any Orlicz function $\psi$ which is submultiplicative on $\mathbb{R}_{+}$because if it were, then $\bar{\varphi}(u)=u^{p} \max (1, u)$ would be equivalent to $\varphi(u)=u^{p} \ln (1+u)$ on $\mathbb{R}_{+}$and this gives a contradiction since $u$ is not equivalent to $\ln (1+u)$ at infinity. This proves our statement in (a).

Concerning the statement in (b), we consider again the Orlicz function $\varphi(u)=u^{p} \ln (1+u)$ for $u \geq 0$ and $p>1$, and define $\psi$ as in Lemma 1. This function $\psi$ satisfies all conditions in statement (b).

Remark 5. It is well-known that any Orlicz function $\varphi$ has an integral representation $\varphi(u)=\int_{0}^{u} \varphi^{\prime}(t) d t$, where $\varphi^{\prime}$ is the right derivative of $\varphi$ and $\varphi(u) \leq u \varphi^{\prime}(u) \leq \varphi(2 u)$ for all $u \geq 0$ (see [8] or [9], [12]). By using these estimates, Ando [3, Lemma 1] (cf. also [12], Theorem 11) has proved that an Orlicz function $\varphi$ is submultiplicative at infinity (on $\mathbb{R}_{+}$) if and only if its right derivative $\varphi^{\prime}$ is submultiplicative at infinity (on $\mathbb{R}_{+}$). Note that in $[7$, Proposition 2] it was observed that if an Orlicz function $\varphi$ satisfies the $\Delta_{2^{-}}$ condition at zero, then $\varphi$ is submultiplicative at zero if and only if its right derivative $\varphi^{\prime}$ is submultiplicative at zero. A similar result can also be proved in the supermultiplicative cases.

An Orlicz function $\varphi$ is supermultiplicative at zero (on $\mathbb{R}_{+}$) if and only if its right derivative $\varphi^{\prime}$ is supermultiplicative at zero (on $\mathbb{R}_{+}$). If $\varphi$ satisfies the $\Delta_{2}$-condition at infinity, then $\varphi$ is supermultiplicative at infinity if and only if its right derivative $\varphi^{\prime}$ is supermultiplicative at infinity.

Let us remark that in [7, Proposition 1 and Lemma 1] there are also proved some extension results for submultiplicative Orlicz functions. In fact it was proved that if an Orlicz function $\varphi$ is submultiplicative at infinity, i.e., on some interval $\left[u_{1}, \infty\right), u_{1}>0$, then $\varphi$ is submultiplicative on $[b, \infty)$ for any $0<b<u_{1}$, and if an Orlicz function $\varphi$ satisfies the $\Delta_{2}$-condition at zero and is submultiplicative at zero; i.e., on some interval $\left[0, u_{0}\right], u_{0}>0$, then $\varphi$ is submultiplicative on $[0, a]$ for any $a>u_{0}$.

By using this technique we can also prove the following complement of the extension results in the supermultiplicativity case: 
Theorem 3. Let $\varphi$ be an Orlicz function.

(a) If $\varphi$ is supermultiplicative at zero, then $\varphi$ is supermultiplicative on the interval $[0, a]$ for any $0<a<\infty$.

(b) If $\varphi$ is supermultiplicative at infinity and $\varphi$ satisfies the $\Delta_{2}$-condition at infinity, then $\varphi$ is supermultiplicative on the interval $[b, \infty)$ for any $0<$ $b<\infty$.

Proof. (a) By assumption, there are positive constants $u_{0}$ and $L$ such that

$$
\varphi(u v) \geq L \varphi(u) \varphi(v) \quad \text { for all } u, v \in\left[0, u_{0}\right] .
$$

Let $0<u_{0}<a<\infty$ and $u, v \in[0, a]$. We consider the following three cases:. $1^{\circ} . u, v \in\left[0, u_{0}\right]$. Then supermultiplicativity holds.

$2^{\circ} . u, v \in\left[u_{0}, a\right]$. By defining

$$
M=\inf \left\{\frac{\varphi(u v)}{\varphi(u) \varphi(v)}: u, v \in\left[u_{o}, a\right]\right\}
$$

we have $0<M<\infty$ and $\varphi(u v) \geq M \varphi(u) \varphi(v)$ for all $u, v \in\left[u_{0}, a\right]$.

$3^{\circ} . u \in\left[0, u_{0}\right]$ and $v \in\left[u_{0}, a\right]$. Then we find that

$$
\varphi(v u) \geq \varphi\left(u_{0} u\right) \geq L \varphi\left(u_{0}\right) \varphi(u)=\frac{L \varphi\left(u_{0}\right)}{\varphi(a)} \varphi(a) \varphi(u) \geq \frac{L \varphi\left(u_{0}\right)}{\varphi(a)} \varphi(v) \varphi(u) .
$$

The proof follows now from the estimates $1^{\circ}-3^{\circ}$ and the constant $K$ in the supermultiplicativity estimate can be taken as

$$
K=\min \left(M, \frac{L \varphi\left(u_{0}\right)}{\varphi(a)}\right) .
$$

(b) The proof is similar to that of (a). (Note only that supermultiplicativity at infinity does not imply in general the $\Delta_{2}$-condition at infinity; so we have assumed it.)

Remark 6. If either both $\varphi$ and $\varphi^{*}$ are submultiplicative Orlicz functions at infinity (at zero or on $\mathbb{R}_{+}$) or $\varphi$ is a submultiplicative as well as a supermultiplicative $N$-function at infinity (at zero or on $\mathbb{R}_{+}$), then $\varphi$ is equivalent to $\varphi_{p}(u)=u^{p}, p>1$, at infinity (at zero or on $\mathbb{R}_{+}$, respectively) (see e.g. [13, Th. 1], [6, Th. 2] or [12, Proposition 12]). 


\section{References}

[1] J. Alexopoulos, De la Vallée Poussin's theorem and weakly compact sets in Orlicz spaces, Quaestiones Math. 17 (1994), 231-248.

[2] T. Ando, On some properties of convex functions, Bull. Acad. Polon. Sci. Sér. Sci. Math. Astronom. Phys. 8 (1960), 413-418.

[3] T. Ando, Certain classes of convex functions, Dokl. Akad. Nauk SSSR 136 (1961), 1007-1010, English transl. in Soviet Math. 2 (1961), 139-142.

[4] A. Böttcher and Yu. I. Karlovich, Submultiplicative functions and spectral theory of Toeplitz operators, Integral Transforms Spec. Funct. 4 (1996), $181-202$.

[5] A. Böttcher and Yu. I. Karlovich, Carleson curves, Muckenhoupt weights and Toeplitz operators, Progress in Mathematics, vol. 154, Birkhäuser, 1997.

[6] C. E. Finol and L. Maligranda, On a decomposition of some functions, Comment. Math. Prace Mat. 30 (1991), 285-291.

[7] H. Hudzik and L. Maligranda, Some remarks on submultiplicative Orlicz functions, Indagationes Math., N. S. 3 (1992), 313-321.

[8] M. A. Krasnoselskii and Yu. B. Rutickii, Convex functions and Orlicz spaces, Groningen, 1961.

[9] L. Maligranda, Indices and interpolation, Dissertationes Math. 234 (1985), 1-49.

[10] L. Maligranda, Orlicz spaces and interpolation, Seminars in Math. 5, Univ. Estadual de Campinas, Campinas SP, Brasil, 1989.

[11] E. Odell and T. Schlumprecht, The distortion problem, Acta Math. 173 (1994), 259-281.

[12] M. M. Rao and Z. D. Ren, Theory of Orlicz spaces, Marcel Dekker, 1991.

[13] D. V. Salekhov, On a property of $N$-functions, Mat. Zametki 4 (1968), 281-290, English transl. in Math. Notes, 4 (1968), 662-667.

[14] M. Zafran, The dichotomy problem for homogeneous Banach algebras, Ann. of Math. 108 (1979), 183-192. 